\section{(6) OPEN ACCESS}

For numbered affiliations see end of article.

\section{Correspondence to} Professor Alexander J Howie, Department of Cellular Pathology, Royal Free Hospital, London NW3 2QG, UK; a.j.howie@ucl.ac.uk

Received 14 February 2014 Revised 20 March 2014 Accepted 21 April 2014 Published Online First 9 May 2014

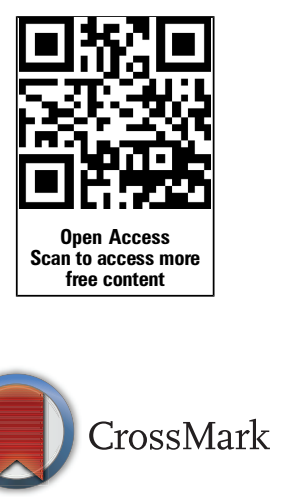

To cite: Owen-Casey MP, Sim R, Cook HT, et al. J Clin Pathol 2014;67:661-666.

\title{
Value of antibodies to free light chains in immunoperoxidase studies of renal biopsies
}

\author{
Mared P Owen-Casey, ${ }_{1}^{1}$ Rosalind Sim, ${ }^{2} \mathrm{H}$ Terence Cook, ${ }^{3}$ Candice A Roufosse, ${ }^{3}$ \\ Julian D Gillmore, ${ }^{4}$ Janet A Gilbertson, ${ }^{4}$ Colin A Hutchison, ${ }^{5}$ Alexander J Howie ${ }^{6}$
}

\begin{abstract}
Aims Because immunoglobulin abnormalities may affect the kidney, investigation of renal biopsies requires immunohistological study of light chains. A problem is that most antibodies to light chains react with whole immunoglobulins as well as free light chains, and there are generally many more whole immunoglobulins than free light chains. The usefulness of antibodies that only detected free light chains was investigated.
\end{abstract}

Methods Antibodies to free light chains were used in an immunoperoxidase method on paraffin sections of 198 renal biopsies, and compared with conventional antibodies against light chains examined by immunofluorescence on 13 frozen sections and by immunoperoxidase on 46 paraffin sections.

Results Immunofluorescence and immunoperoxidase were concordant on 10 of 13 biopsies.

Immunofluorescence detected slight deposition of light chains in three biopsies not shown by immunoperoxidase, of undetermined clinical significance. Using immunoperoxidase, the free light chain antibodies were more sensitive than conventional antibodies, giving much cleaner staining and better detection of deposits in AL amyloid, light chain deposition disease and cryoglobulinaemic glomerulonephritis. The free light chain antibodies showed discordance or ambiguity between immunohistological and clinical findings in seven (4\%) of 185 patients with known immunoglobulin status. These included two of 28 cases of AL amyloid that showed no light chain deposition. The method was not designed for detection of light chain restriction in neoplastic plasma or lymphoplasmacytic cells.

Conclusions Polyclonal antibodies to free light chains are an improvement on conventional antibodies in immunoperoxidase study of paraffin sections of renal biopsies and are useful in everyday practice.

\section{INTRODUCTION}

Abnormal immunoglobulin light chains may have various effects on the kidney, especially cast nephropathy, $\mathrm{AL}$ amyloidosis or deposition disease, although they may have no detectable effects. ${ }^{1-4}$ Any study of light chains in renal biopsies, by immunofluorescence on frozen or paraffin sections or immunoperoxidase on paraffin sections, has disadvantages, including the specially collected material required for frozen sections, impermanence of fluorescence, and pretreatment usually needed for paraffin sections, but most antibodies give comparable findings between frozen and paraffin sections. ${ }^{5-9}$

A problem is the specificity of antibodies against light chains. Most polyclonal antibodies react with light chains both free and bound to heavy chains, but usually react preferentially with whole immunoglobulins, because normal mean plasma concentrations of free light chains and whole immunoglobulins are about $23 \mathrm{mg} / \mathrm{L}^{10}$ and $19 \mathrm{~g} / \mathrm{L}^{11}$ one thousand times different. Monoclonal antibodies may be specific for a free light chain, but the epitope recognised may not be expressed or accessible in all light chains. ${ }^{12}$

Polyclonal antibodies specific for free light chains are available,${ }^{10}$ with applications in measurement of plasma light chains and diagnosis and management of clinical immunoglobulin disorders. ${ }^{13}$ Our study assessed the value of these antibodies in immunohistology of paraffin sections of renal biopsies, including comparison with conventional antibodies.

\section{MATERIALS AND METHODS Antibodies}

Sheep antibodies to human free $\kappa$ and $\lambda$ light chains were supplied by The Binding Site, Birmingham, UK. Rabbit antibodies to $\kappa$ and $\lambda$ (A0191, A0193), mouse antibody to serum amyloid A (M0759) and fluorescein-conjugated antibodies to $\kappa$ and $\lambda$ (F0198, F0199) were obtained from Dako.

\section{Immunostaining}

For immunoperoxidase, endogenous peroxidase was blocked on dewaxed $3 \mu \mathrm{m}$ paraffin sections by hydrogen peroxide. After preliminary experiments, optimum antigen retrieval for sheep antibodies was digestion with protease type 24 (Sigma, P8038), $0.05 \% \mathrm{w} / \mathrm{v}$ in phosphate buffered saline $\mathrm{pH} 7.2$, at $37^{\circ} \mathrm{C}$, initially for $45 \mathrm{~min}$, with microscopic study of completeness of digestion and further digestion if necessary. ${ }^{5}$ Antibodies were applied at 1:400 (к) or $1: 200(\lambda)$ for $45 \mathrm{~min}$, then rabbit anti-sheep immunoglobulins, peroxidase-conjugated (Dako, P0163), at 1:100, for $45 \mathrm{~min}$, followed by diaminobenzidine and hydrogen peroxide, and haematoxylin. Various methods of antigen retrieval for rabbit antibodies were tried, including no pretreatment, protease digestion, trypsin digestion, microwave heating, pressure cooking, and heating for $30 \mathrm{~min}$ in antigen retrieval solution $\mathrm{pH} 9.0$ provided in a kit for an automated method (Bondmax: Leica Microsystems, Milton Keynes, UK). Most pretreatments matched automated antigen retrieval, but protease abolished immunostaining and no pretreatment gave weak immunostaining. Antibodies were applied at 1:20 000, followed by Bondmax anti-rabbit Poly-HRP-IgG, peroxidase substrate and counterstain. Amyloid A antibody (1:500) was used without pretreatment followed by Bondmax antimouse antibody. Frozen sections were covered with 
fluoresceinated antibodies and examined by fluorescence microscopy.

Thirty-two patients were referred to the National Amyloidosis Centre (NAC). Their renal biopsies were independently examined with the rabbit antibodies to light chains without antigen retrieval, and with antibodies to amyloid $\mathrm{A}$ and if necessary to other potential amyloid precursors. ${ }^{14}$

\section{Renal biopsies}

Thirteen biopsies examined by immunofluorescence on frozen sections were studied using the free light chain antibodies on paraffin sections, as were another 185 paraffin-embedded biopsies. These included 46 biopsies used to compare the free light chain and rabbit antibodies, 32 at NAC and 14 others. Immunostained paraffin sections were examined without knowledge of clinical features or original reports of light chain findings. Diagnoses were made in usual ways. ${ }^{15}$ Conventional summary statistics (sensitivity, specificity and CIs of differences in proportions) were calculated.

\section{Evidence of immunoglobulin disorders}

Apart from three cases studied by immunofluorescence, all others had evidence for or against a disorder of immunoglobulins. Evidence was any of these: a serum paraprotein; a cryoglobulin; an abnormal ratio of serum free $\kappa$ to $\lambda$ concentrations ${ }^{10}$; or a monoclonal (Bence Jones) light chain in urine. The Results mostly concentrate on the 185 biopsies, selected because there was adequate information about immunoglobulin status of the patients, 110 with an immunoglobulin abnormality and 75 without. Free light chain immunostaining was more likely to be done if an abnormality was known or suspected or was in the differential diagnosis.

\section{RESULTS}

Comparison of immunofluorescence and immunoperoxidase

Ten of 13 biopsies showed agreement between the methods, with AL amyloid (5), no light chain deposition (3), and deposition disease (2). The other three showed no free light chain deposition, and only faint deposition on immunofluorescence, one of $\kappa$ in tubular basement membranes with slight chronic damage, one of $\kappa$ in glomerular basement membranes but no structural glomerular abnormality, and one of more $\lambda$ than $\kappa$ in tubular basement membranes but no clinical immunoglobulin abnormality.

\section{Comparison of different antibodies using immunoperoxidase}

The 14 non-NAC biopsies comparing free light chain and rabbit antibodies had cast nephropathy (3), amyloid (3, two AL, one AA), no immunoglobulin abnormality (3), renal infiltration by lymphoplasmacytic lymphoma or myeloma (2), cryoglobulinaemic glomerulonephritis (2, both type II) and deposition disease (1). Rabbit antibodies after automated antigen retrieval showed heavy staining of tubular epithelium, interstitial tissues and blood, but background staining was much lighter without pretreatment (figures 1, 2). After automated antigen retrieval, deposits of light chains in $\mathrm{AL}$ amyloid, cryoglobulinaemia and deposition disease were impossible to distinguish from the background, and without pretreatment, immunostaining for light chains was weak and only seen in one case, with AL amyloid. Deposits were easily detected with free light chain antibodies, with clean background and no blood in vessels (figures 3, 4).

Both types of antibody showed a preponderance of one light chain in casts and tubular epithelium in cast nephropathy. Light

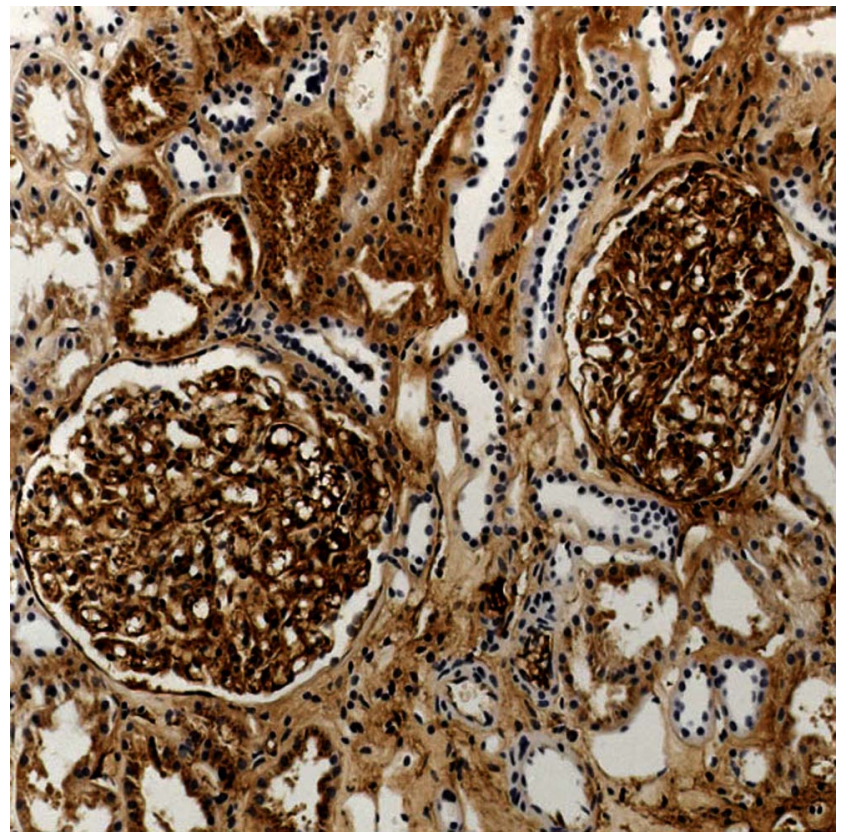

Figure 1 Renal biopsy containing amyloid in glomeruli in a patient with a $\kappa$ paraprotein. Immunostaining with the rabbit antibody to $\kappa$ light chains after automated antigen retrieval shows extensive staining but no detectable selective staining of amyloid deposits.

chain restriction was identified in the myelomatous infiltrate only with rabbit antibodies, but with neither antibody in the lymphoplasmacytic lymphoma. Comparison of the antibodies, including findings in NAC (below), is in table 1.

\section{Amyloid}

With free light chain antibodies, 25 of 41 biopsies showed AL amyloid $(21 \lambda$, four $\kappa)$, with concordance between clinical and

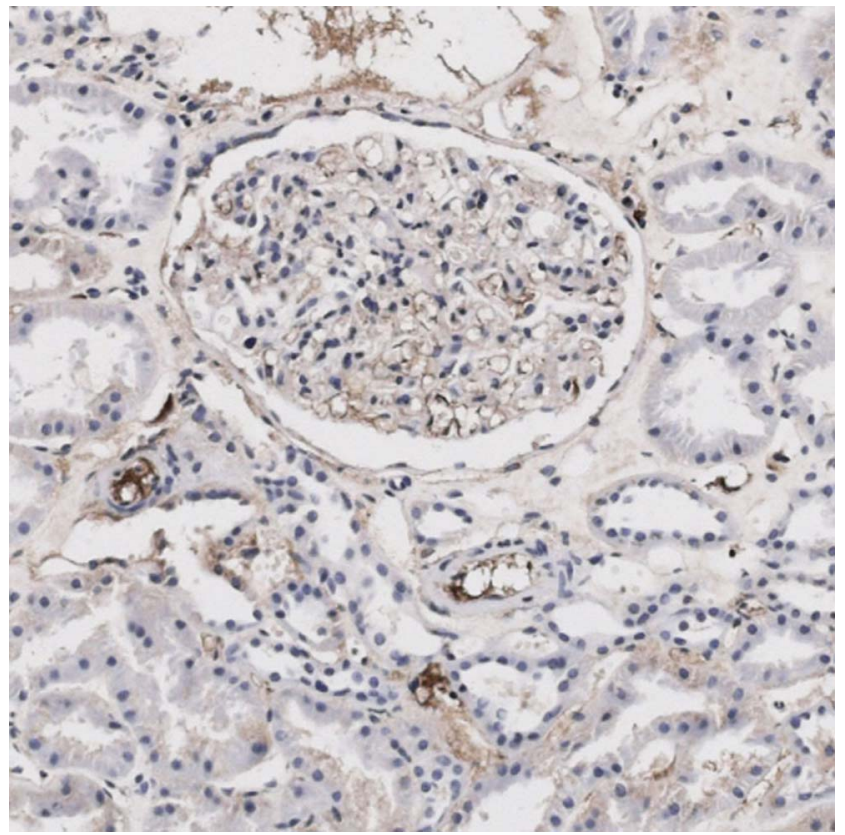

Figure 2 The same renal biopsy as in figure 1. Immunostaining with the rabbit antibody to $\kappa$ light chains without pretreatment shows light background staining but no detectable staining of amyloid deposits. 


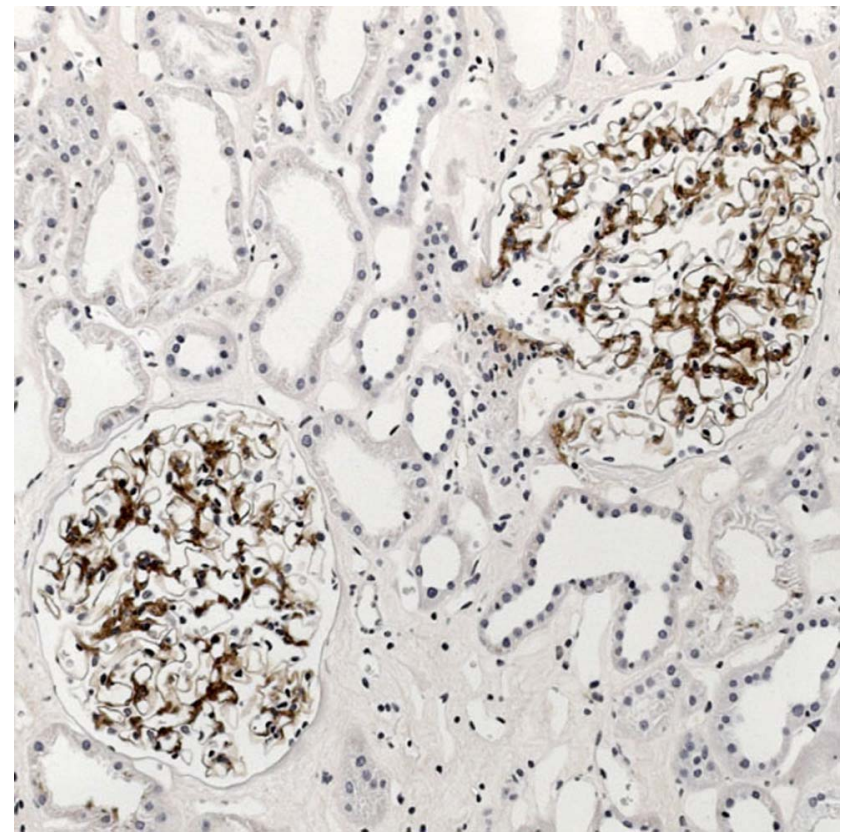

Figure 3 The same renal biopsy as in figure 1. Immunostaining with the sheep antibody to free $\kappa$ light chains shows selective staining of amyloid deposits in glomeruli.

immunoperoxidase findings (tables 2, 3). NAC confirmed $\lambda$ AL amyloid in 13 of $17 \lambda$ cases and $\kappa$ AL amyloid in one of two $\kappa$ cases by immunohistology, and in four and one, respectively, from overall evidence.

Eight biopsies showed AA amyloid and three nonAL, nonAA amyloid with no free light chain immunostaining, concordant with no immunoglobulin disorder. NAC confirmed five cases of AA amyloid by immunohistology, and identified two nonAL, nonAA cases as LECT2 amyloid and one as fibrinogen A $\alpha$ amyloid. Another case had no clinical immunoglobulin

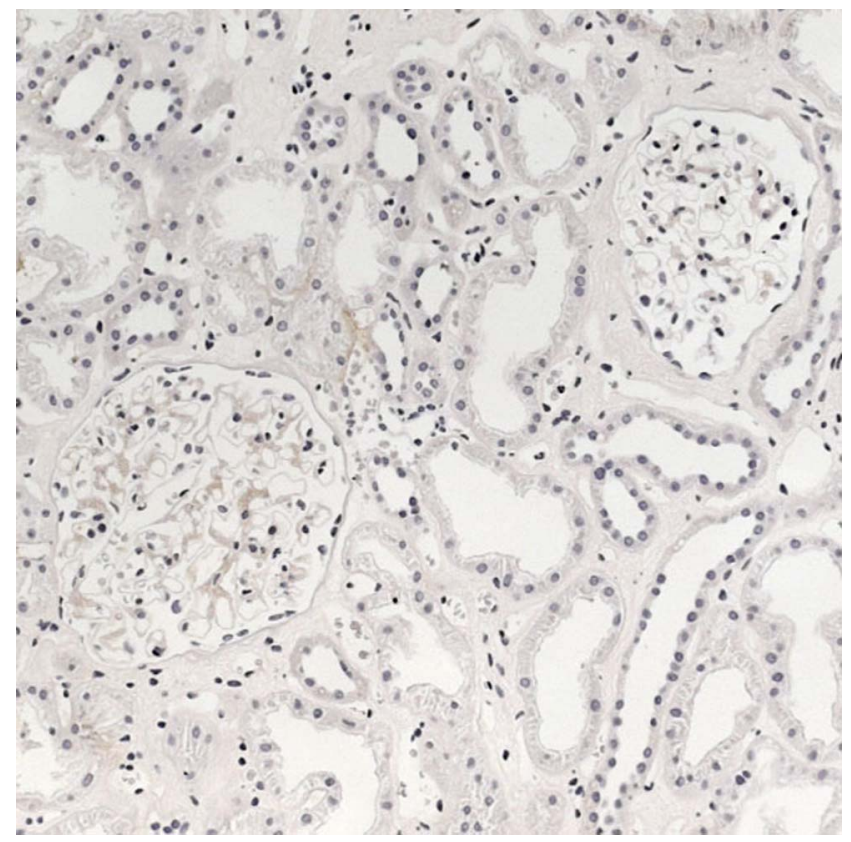

Figure 4 The same renal biopsy as in figure 1. Immunostaining with the sheep antibody to free $\lambda$ light chains shows no staining.
Table 1 Comparison of diagnostic accuracy of antibodies to free light chains and conventional antibodies to light chains in 46 renal biopsies, compared with final diagnosis of any renal abnormality related to light chain disorders, including neoplastic infiltration

\begin{tabular}{ll}
\hline $\begin{array}{l}\text { Antibodies to free light chains } \\
\text { Deposition }\end{array}$ & No deposition \\
\hline 29 & 17 \\
Abnormality/no abnormality & Abnormality/no abnormality \\
$28 / 1^{*}$ & $4 t / 13$ \\
Sensitivity $88 \%$, specificity $93 \%$ & \\
\hline
\end{tabular}

\section{Conventional antibodies to light chains}

Deposition

No deposition

20

Abnormality/no abnormality

20/0
26

Abnormality/no abnormality $12 \ddagger / 14$
Sensitivity $63 \%$, specificity $100 \%$

Difference in sensitivity (free light chain antibodies - conventional antibodies) $=25 \%$ (95\% Cl 9\% to $41 \%$ ); difference in specificity (free light chain antibodies conventional antibodies $)=-7 \%(95 \% \mathrm{Cl}-14 \%$ to $0 \%)$.

${ }^{*} \kappa$ \& AA deposition in AA amyloid.

†No deposition in AL amyloid (2), neoplastic infiltration (2).

¥No deposition in AL amyloid (8), cryoglobulinaemic glomerulonephritis (2), light chain deposition disease (1), neoplastic infiltration (1).

abnormality, but amyloid deposits reacted with antibodies to amyloid A and free $\kappa$. NAC diagnosed AA amyloid immunohistologically.

The other four cases had a clinical immunoglobulin abnormality. One with a $\kappa$ paraprotein showed deposition of both free $\kappa$ and amyloid A. NAC showed no deposition on immunohistology but considered this $\kappa \mathrm{AL}$ amyloid on overall evidence. In the other three, no free light chain deposition was detected. One with a $\lambda$ Bence Jones protein was confirmed $\lambda$ AL amyloid immunohistologically at NAC. One with a $\lambda$ paraprotein had no immunohistological deposition at NAC but was considered $\lambda \mathrm{AL}$ amyloid on overall evidence. In one case with a $\kappa$ paraprotein, the amyloid reacted only with amyloid A antibody. AA amyloid was confirmed by NAC.

\section{Other conditions}

Light chain cast nephropathy

Findings were concordant between immunostaining for free light chains and immunoglobulin abnormalities in 32 of 33 cases $(26 \kappa$, six $\lambda)$, with definite preponderance of one light chain in casts and tubular epithelial cells (figures 5,6 ). In the

Table 2 Light chain immunohistological findings in 41 renal biopsies containing amyloid

\begin{tabular}{|c|c|c|c|}
\hline & AL amyloid & $\begin{array}{l}\text { AA } \\
\text { amyloid }\end{array}$ & $\begin{array}{l}\text { Other } \\
\text { amyloid }\end{array}$ \\
\hline Final diagnosis & $28(23 \lambda, 5 \kappa)$ & 10 & 3 \\
\hline $\begin{array}{l}\text { Free light chain } \\
\text { antibodies } n=41\end{array}$ & $\begin{array}{l}21 \lambda+v e, 2 \lambda-v e, 4 \kappa+v e, 1 \\
\kappa \& A A+v e(25 / 28(89 \%) \\
\text { unambiguously diagnostic) }\end{array}$ & $\begin{array}{l}9-v e, 1 \kappa \\
\& A A+v e\end{array}$ & $3-v e$ \\
\hline $\begin{array}{l}\text { Conventional light } \\
\text { chain antibodies } \\
n=32\end{array}$ & $\begin{array}{l}14 \lambda+\mathrm{ve}, 5 \lambda-\mathrm{ve}, 1 \kappa+\mathrm{ve}, 2 \\
\kappa-\mathrm{ve}(15 / 22(68 \%) \\
\text { diagnostic) }\end{array}$ & $7-$ ve & $3-v e$ \\
\hline
\end{tabular}

For AL amyloid, difference in proportion unambiguously diagnostic (antibodies to fre light chains - conventional antibodies to light chains) $=21 \%$ (95\% Cl $2 \%$ to $39 \%$ ). 
Table 3 Summary of immunohistological findings with antibodies to free light chains in 185 renal biopsies and immunoglobulin status of patients (as defined in materials and methods)

\begin{tabular}{|c|c|c|c|c|}
\hline & $\begin{array}{l}\text { Light chain deposition and } \\
\text { immunoglobulin } \\
\text { abnormality }\end{array}$ & $\begin{array}{l}\text { No light chain deposition and } \\
\text { no immunoglobulin } \\
\text { abnormality }\end{array}$ & $\begin{array}{l}\text { No light chain deposition } \\
\text { and immunoglobulin } \\
\text { abnormality }\end{array}$ & $\begin{array}{l}\text { Light chain deposition and } \\
\text { no immunoglobulin } \\
\text { abnormality }\end{array}$ \\
\hline Amyloid $n=41$ & $26\left(1^{*}\right)$ & 11 & $3\left(1 \dagger, 2^{*}\right)$ & $1^{*}$ \\
\hline $\begin{array}{l}\text { Light chain cast nephropathy } \\
n=33\end{array}$ & 32 & - & $1^{*}$ & - \\
\hline $\begin{array}{l}\text { Light chain deposition disease } \\
\mathrm{n}=6\end{array}$ & 6 & - & 0 & - \\
\hline Cryoglobulinaemia $n=6$ & 4 & - & $2\left(1 \dagger, 1^{*}\right)$ & - \\
\hline Miscellaneous $n=3$ & 3 & - & 0 & - \\
\hline Neoplastic infiltration $n=2$ & 0 & - & $2+$ & - \\
\hline $\begin{array}{l}\text { No evidence of renal effects of } \\
\text { immunoglobulin abnormality } \\
n=31\end{array}$ & 0 & - & $31+$ & - \\
\hline $\begin{array}{l}\text { No immunoglobulin abnormality } \\
\text { (excluding amyloid cases) } n=63\end{array}$ & - & 62 & - & $1^{*}$ \\
\hline
\end{tabular}

${ }^{*}$ Considered discordant.

†Not considered discordant, as discussed in text.

discordant case, there was a $\kappa$ paraprotein and a few casts showed anomalous colours with Congo red as found in some cases of cast nephropathy, without definite preponderance of $\kappa$ on immunostaining.

\section{Light chain deposition disease}

Findings were concordant in all six cases of deposition disease (five $\kappa$, one $\lambda$ ), with glomerular deposition of the appropriate light chain, and often deposition in tubular basement membranes (figure 7).

Other renal effects of immunoglobulin abnormalities

In four of 11 cases, free light chain immunostaining did not show a preponderance of one light chain. Two were kidneys

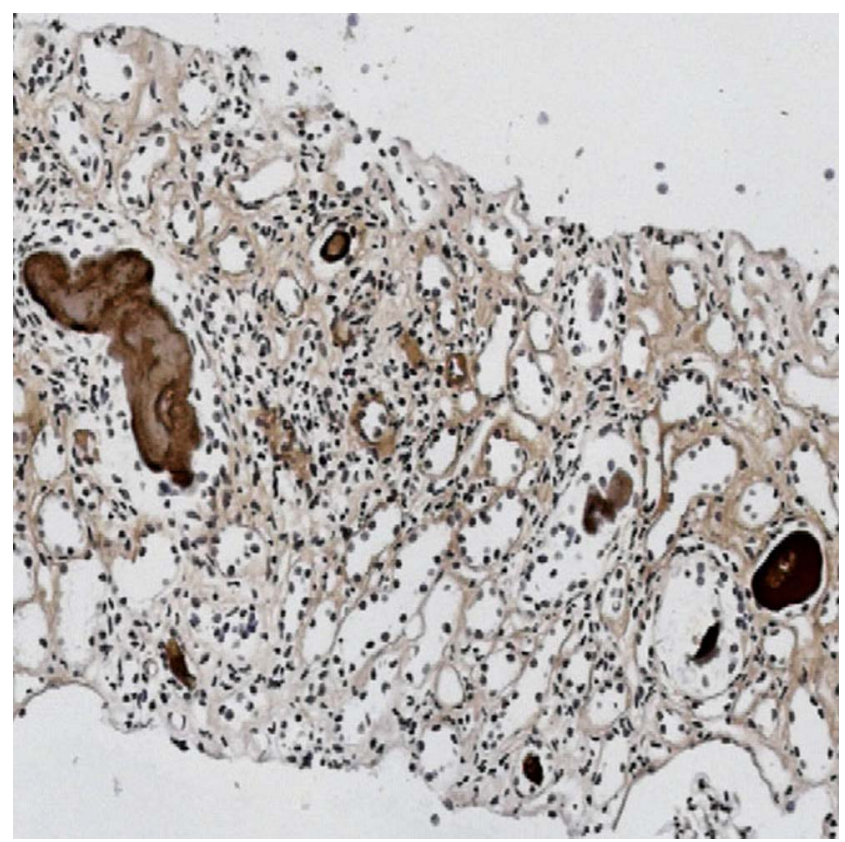

Figure 5 Renal biopsy showing light chain cast nephropathy in a patient with an $\lg A \lambda$ paraprotein. Immunostaining with the sheep antibody to free $\lambda$ light chains shows deposition in casts. infiltrated by myeloma and lymphoplasmacytic lymphoma, and two were cases of cryoglobulinaemia (one type II, one type III). There was concordance in the other seven cases, all $\kappa$, four cryoglobulinaemic glomerulonephritis (one type I, three type II) (figure 8), two tubulointerstitial nephritis with light chains in tubular basement membranes, and one with crystalline deposits of light chains in podocytes.

No evidence of renal effects of an immunoglobulin abnormality In 31 cases, there was no detectable effect of a paraprotein in the kidney and no immunohistological disproportion between $\mathrm{K}$ and $\lambda$. The commonest diagnoses were diabetic glomerulopathy (6), membranous nephropathy (4) and chronic ischaemic damage (4).

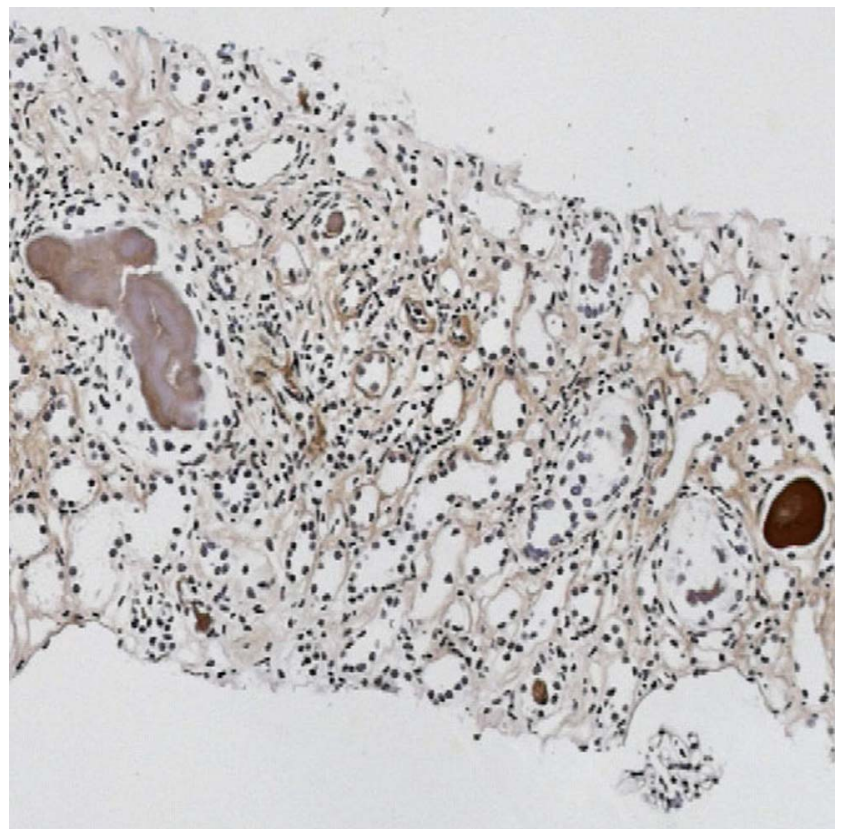

Figure 6 The same renal biopsy as in figure 5. Immunostaining with the sheep antibody to free $\kappa$ light chains shows less deposition of $\kappa$ than $\lambda$ (figure 5 ). 


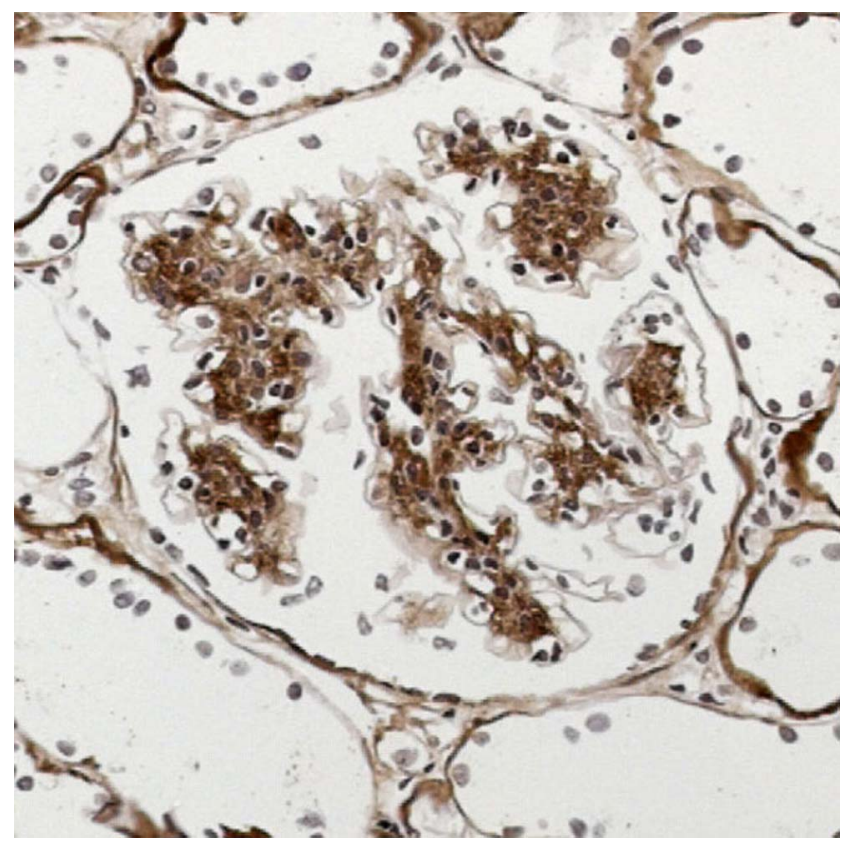

Figure 7 Renal biopsy showing light chain deposition disease in a patient with a $\kappa$ paraprotein. Immunostaining with the sheep antibody to free $\kappa$ light chains shows deposition in mesangium and tubular basement membranes. There was no deposition of free $\lambda$ light chains.

\section{No immunoglobulin abnormality}

Excluding 12 amyloid cases, 63 biopsies were from patients without an immunoglobulin abnormality as defined above, although many had immunoglobulin deposits, shown by antibodies to heavy chains. In 62 there was no disproportion between $\kappa$ and $\lambda$ on immunohistology. One showed subendothelial membranoproliferative glomerulonephritis with more $\kappa$ than $\lambda$ in glomerular deposits, without evidence of a monoclonal gammopathy.

\section{DISCUSSION}

Immunofluorescence on frozen sections and immunoperoxidase or immunofluorescence on paraffin sections rarely show complete concordance in detection of light chain deposition. Which method gives the correct or more clinically relevant finding is undetermined. ${ }^{6-9}$ The discrepancies in our study were minor and of uncertain clinical significance.

Comparison on paraffin sections between free light chain and conventional antibodies showed that differences were due to the antibodies, not pretreatments. The free light chain antibodies did not detect whole immunoglobulins and were more sensitive than conventional antibodies (table 1). Light chains in deposits were readily differentiated from background. The free light chain antibodies were better at typing AL amyloid, giving stronger and generally unequivocal staining (figures 3 , 4; table 2). Two of 28 cases (7\%) of AL amyloid were missed, although to different extents all antibodies to light chains miss cases of $\mathrm{AL}$ amyloid, up to $35 \%$ in one series. ${ }^{14} 1617$ Some cases of AL amyloid will still need other investigations for definitive diagnosis, such as laser microdissection of sections and mass spectrometry. ${ }^{17} 18$ Reactivity of amyloid with multiple antibodies has been reported, for instance in 34\% of biopsies with AA amyloid, ${ }^{19}$ and two of our 41 amyloid cases $(5 \%)$ showed ambiguous immunostaining for both free $\kappa$ and amyloid A. Four amyloid cases (10\%) therefore showed

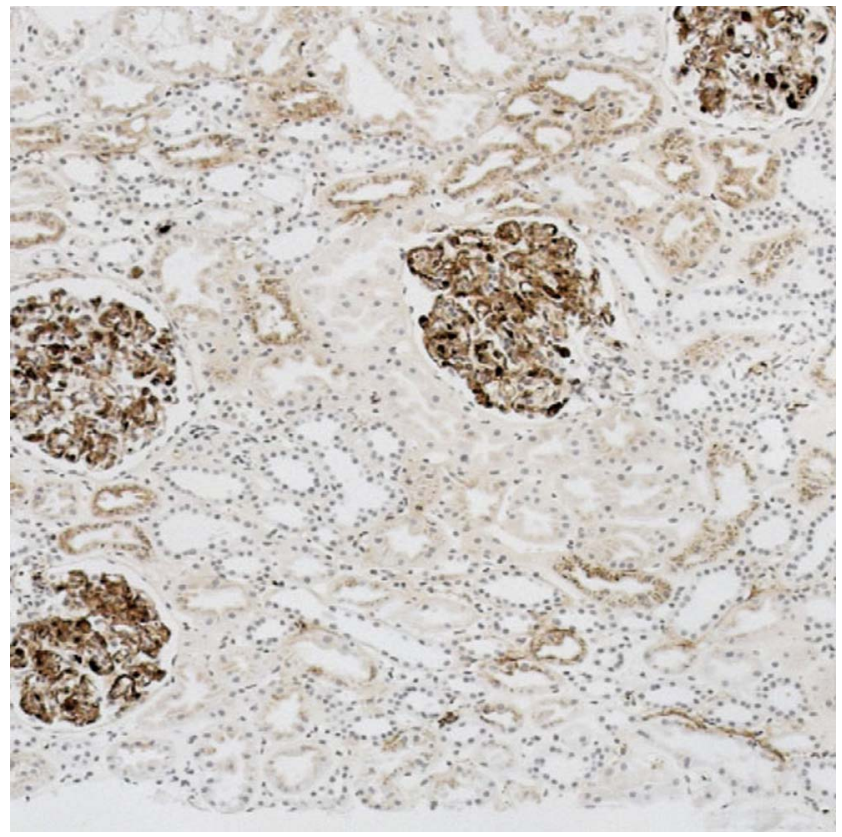

Figure 8 Renal biopsy showing type II cryoglobulinaemic glomerulonephritis in a patient with an $\lg \mathrm{M} \kappa$ paraprotein. Immunostaining with the sheep antibody to free $\kappa$ light chains shows extensive deposition in glomeruli. There was no deposition of free $\lambda$ light chains.

discordance between clinical and immunohistological findings, overlooking one case with a paraprotein but confirmed to be AA amyloid.

The free light chain antibodies were useful in confirmation of deposition disease, with complete concordance, and usually of cryoglobulinaemic glomerulonephritis (figures 7, 8). The discordant case of type II cryoglobulinaemia, with mixed monoclonal IgM and polyclonal IgG, may have had insufficient excess of free light chains to be detectable. In type III cryoglobulinaemia, with polyclonal deposits, no excess of one free light chain would be expected, and was not considered discordant. There was little difference between free light chain and conventional antibodies in detection of light chains in cast nephropathy. The failure of free light chain antibodies to show an excess of one light chain in one of 33 cases (3\%) may be because in virtually every case of cast nephropathy both light chains are found (figures 5,6), and a small excess of one may be undetectable.

The free light chain method was developed to detect deposits rather than intracellular or surface immunoglobulins, and neoplastic infiltrates can be investigated in other ways. ${ }^{20}$ There was no deposition of free light chains in 32 cases with a monoclonal gammopathy but without diagnosable renal effects, including one case of AA amyloid $(29 \%$ of those with immunoglobulin abnormalities). Similarly, $27 \%$ of patients with myeloma ${ }^{3}$ and $63 \%$ with a monoclonal gammopathy ${ }^{1}$ had no evidence of renal lesions related to a paraprotein. Accordingly, only seven of 185 cases (4\%) were considered discordant: four amyloid, one cryoglobulinaemia, one light chain cast nephropathy, and one glomerulonephritis with excess $\kappa$ deposition but no gammopathy.

Polyclonal free light chain antibodies are of practical use in the study of renal biopsies and have advantages over conventional light chain antibodies. Methods are similar and overall costs are also likely to be similar. 


\section{Take home messages}

- Polyclonal antibodies to free light chains are an improvement on conventional antibodies to light chains in the immunoperoxidase study of paraffin sections of renal biopsy specimens, giving a cleaner background and sharper discrimination of sites of deposition.

- These antibodies are more sensitive than conventional antibodies in the detection of cases of immunological disorders in the kidney such as AL amyloid, light chain deposition disease and cryoglobulinaemic glomerulonephritis.

\section{Author affiliations}

${ }^{1}$ Department of Histopathology, Wrexham Maelor Hospital, Wrexham, UK

${ }^{2}$ Department of Cellular Pathology, Royal Free Hospital, London, UK

${ }^{3}$ Department of Histopathology, Imperial College School of Medicine, Hammersmith Hospital, London, UK

${ }^{4}$ UCL Division of Medicine, National Amyloidosis Centre, Royal Free Hospital, London, UK

${ }^{5}$ Department of Nephrology, Hawke's Bay District Health Board, Hastings, New Zealand

${ }^{6}$ Department of Pathology, University College London, London, UK

Acknowledgements We are grateful to The Binding Site Group Ltd (UK) (http:// www.thebindingsite.com/) for the antibodies to free light chains.

Contributors MPOC and AJH planned the study, analysed the immunoperoxidase findings and wrote initial drafts of the paper. RS developed and performed the free light chain immunostaining. HTC and CAR analysed the immunofluorescence findings. JDG and JAG performed and analysed the immunostaining at NAC. CAH collaborated with The Binding Site Group Ltd (UK) to obtain the antibodies to free light chains. All authors contributed to revisions of the manuscript and approved the final version.

Competing interests $\mathrm{CAH}$ reports grants and personal fees from The Binding Site Group Ltd (UK) outside the submitted work.

Ethics approval NRES Committee London (Hampstead).

Provenance and peer review Not commissioned; externally peer reviewed.

Open Access This is an Open Access article distributed in accordance with the Creative Commons Attribution Non Commercial (CC BY-NC 3.0) license, which permits others to distribute, remix, adapt, build upon this work non-commercially, and license their derivative works on different terms, provided the original work is properly cited and the use is non-commercial. See: http://creativecommons.org/ licenses/by-nc/3.0/

\section{REFERENCES}

1 Paueksakon P, Revelo MP, Horn RG, et al. Monoclonal gammopathy: significance and possible causality in renal disease. Am J Kidney Dis 2003;42:87-95.

2 Basnayake K, Stringer SJ, Hutchison CA, et al. The biology of immunoglobulin free light chains and kidney injury. Kidney Int 2011;79:1289-301.

3 Nasr SH, Valeri AM, Sethi S, et al. Clinicopathologic correlations in multiple myeloma: a case series of 190 patients with kidney biopsies. Am J Kidney Dis 2012; 59:786-94.

4 Leung N, Bridoux F, Hutchison CA, et al. Monoclonal gammopathy of renal significance: when MGUS is no longer undetermined or insignificant. Blood 2012;120:4292-5.

5 Howie AJ, Gregory J, Thompson RA, et al. Technical improvements in the immunoperoxidase study of renal biopsy specimens. J Clin Pathol 1990;43:257-9.

6 Molne J, Breimer ME, Svalander CT. Immunoperoxidase versus immunofluorescence in the assessment of human renal biopsies. Am J Kidney Dis 2005; 45:674-83

7 Nasr SH, Galgano SJ, Markowitz GS, et al. Immunofluorescence on pronase-digested paraffin sections: a valuable salvage technique for renal biopsies. Kidney Int 2006;70:2148-51.

8 Picken MM. Current practice in amyloid detection and typing among renal pathologists. Amyloid 2011;18(Suppl 1):73-5.

9 Shi S, Zhang P, Cheng Q, et al. Immunohistochemistry of deparaffinised sections using antigen retrieval with microwave combined pressure cooking versus immunofluorescence in the assessment of human renal biopsies. J Clin Pathol 2013;66:374-80.

10 Bradwell AR, Carr-Smith HD, Mead GP, et al. Highly sensitive, automated immunoassay for immunoglobulin free light chains in serum and urine. Clin Chem 2001;47:673-80.

11 Marshall WJ, Bangert SK, Lapsley M, eds. Clinical chemistry. 7th edn. Edinburgh: Mosby, 2012:229.

12 Hutchison CA, Cockwell P, Cook M. Diagnostic accuracy of monoclonal antibody based serum immunoglobulin free light chain immunoassays in myeloma cast nephropathy. BMC Clin Pathol 2012;12:12.

13 Hutchison CA, Plant T, Drayson $M$, et al. Serum free light chain measurement aids the diagnosis of myeloma in patients with severe renal failure. BMC Nephrol 2008;9:11.

14 Gilbertson JA, Hunt T, Hawkins PN. Amyloid typing: experience from a large referral centre. In: Picken MM, Dogan A, Herrera GA, eds. Amyloid and related disorders: surgical pathology and clinical correlations. New York: Springer, 2012:231-8.

15 Jennette JC, Olson JL, Schwartz MM, et al., eds. Heptinstall's pathology of the kidney. 6th edn. Philadelphia: Lippincott Williams and Wilkins, 2006.

16 Novak L, Cook WJ, Herrera GA, et al. AL-amyloidosis is underdiagnosed in renal biopsies. Nephrol Dial Transplant 2004;19:3050-3.

17 Said SM, Sethi S, Valeri AM, et al. Renal amyloidosis: origin and clinicopathologic correlations of 474 recent cases. Clin J Am Soc Nephrol 2013;8:1515-23.

18 Sethi S, Vrana JA, Theis JD, et al. Laser microdissection and mass spectrometry-based proteomics aids the diagnosis and typing of renal amyloidosis. Kidney Int 2012;82:226-34

19 Verine J, Mourad N, Desseaux K, et al. Clinical and histological characteristics of renal AA amyloidosis: a retrospective study of 68 cases with a special interest to amyloid-associated inflammatory response. Hum Pathol 2007;38:1798-809.

20 Marshall-Taylor CE, Cartun RW, Mandich D, et al. Immunohistochemical detection of immunoglobulin light chain expression in B-cell non-Hodgkin lymphomas using formalin-fixed, paraffin-embedded tissues and a heat-induced epitope retrieval technique. Appl Immunohistochem Mol Morphol 2002;10:258-62. 